This is an electronic reprint of the original article. This reprint may differ from the original in pagination and typographic detail.

Author(s): Dong, G X; Wang, Xiaobao; Liu, H.L.; Xu, F.R.

Title: $\quad$ Collectivity of neutron-rich magnesium isotopes investigated by projected shell model calculations

Year: $\quad 2013$

Version:

Please cite the original version:

Dong, G. X., Wang, X., Liu, H.L., \& Xu, F.R. (2013). Collectivity of neutron-rich magnesium isotopes investigated by projected shell model calculations. Physical Review C, 88(2), Article 024328. https://doi.org/10.1103/PhysRevC.88.024328

All material supplied via JYX is protected by copyright and other intellectual property rights, and duplication or sale of all or part of any of the repository collections is not permitted, except that material may be duplicated by you for your research use or educational purposes in electronic or print form. You must obtain permission for any other use. Electronic or print copies may not be offered, whether for sale or otherwise to anyone who is not an authorised user. 


\title{
Collectivity of neutron-rich magnesium isotopes investigated by projected shell model calculations
}

\author{
G. X. Dong, ${ }^{1}$ X. B. Wang, ${ }^{2}$ H. L. Liu, ${ }^{3}$ and F. R. $X u^{1, *}$ \\ ${ }^{1}$ State Key Laboratory of Nuclear Physics and Technology, School of Physics, Peking University, Beijing 100871, China \\ ${ }^{2}$ Department of Physics, P.O. Box 35 (YFL), University of Jyväskylä, FI-40014 Jyväskylä, Finland \\ ${ }^{3}$ Department of Applied Physics, Xian Jiaotong University, Xian 710049, China \\ (Received 5 June 2013; revised manuscript received 11 August 2013; published 30 August 2013)
}

\begin{abstract}
The abnormally large collectivity of neutron-rich magnesium isotopes in the "island of inversion" has not been well understood. It has been commented that the unexpectedly large deformations observed in the magnesium isotopes are attributed to the neutron $f_{7 / 2}$ intruder orbits involved remarkably even in the ground states, which points to nuclear force directly. Recently, a new isospin-dependent Nilsson potential was suggested to improve the calculations of the ground states of magnesium isotopes. With the improved Nilsson potential, in the present work we investigate the collectivity of excited states by using the projected shell model. To avoid the collapse of the BCS pairing, which occurs in weak pairing, we improved pairing calculations by using the Lipkin-Nogami approach. The collectivity and the erosion of the $N=20$ shell in neutron-rich magnesium isotopes are discussed by calculating spectra and electric quadrupole transitions. The gyromagnetic factors are calculated and compared with existing data to get insight into the configurations of collective states at different spins. A two-quasineutron $K^{\pi}=6^{+}$state in ${ }^{38} \mathrm{Mg}$ is predicted to be a possible isomer with an excitation energy lower than the $I^{\pi}=6^{+}$ member of the ground-state band.
\end{abstract}

DOI: 10.1103/PhysRevC.88.024328

PACS number(s): 21.10.Re, 21.10.Ky, 21.60.Cs, 27.30.+t

\section{INTRODUCTION}

Whether the magic numbers established in the valley of stable nuclei exist in exotic nuclei is still an open question [1]. The first indication of the vanishing of shell closures was revealed around the neutron magic number $N=20$ [1-3]. It has been pointed out that the $N=20$ gap is no longer large enough to hinder the cross-shell excitations for magnesium isotopes [4], and a considerable prolate deformation was observed for the ground state of ${ }^{32} \mathrm{Mg}$ through the measurements of the $E 2$ transition strength [4-6] and rms charge radii [7]. The lowlying excited states contain significant intruder configurations, i.e., configurations outside the $s d$ shell $[4,8-10]$. The normal and intruder configurations are even inverted in energies [10]. The neighboring nuclei with the inversion form an "island of inversion" [11].

There have been many theoretical efforts to understand the anomalously large collectivity of magnesium isotopes, e.g., by shell models [12-17] and mean-field approaches [18-20]. The shell model is advantageous in the study of interaction between normal and intruder configurations, while the mean-field theory is more straightforward for describing collective properties. It has been shown that the projected shell model [21] is a bridge connecting these two models and can provide useful information about the exciting mechanism and configurations of rotational states.

The evolution of shell closures has attracted great interest from both theorists and experimentalists. It was pointed out that the erosion of the magic numbers established near stable nuclei can be caused by the neutron-proton interaction [14,22-24]. In the present work, we investigate the structures and collectivities of magnesium isotopes using the projected

*frxu@pku.edu.cn shell model (PSM) which is built up by the spatial-symmetryconserved multi-quasiparticle (multi-qp) configurations projected from the Nilsson model [25]. However, the standard Nilsson parameters (which are isospin independent and were usually fitted to experimental properties of nuclei near the stability line) are not able to give a proper description of deformations of neutron-rich magnesium isotopes. Recently, a special set of the parameters of the Nilsson potential was suggested to describe the ground-state deformations of the chain of magnesium isotopes [26]. It was emphasized that the isospin dependence should be added to the Nilsson parameters in order to take the effect of the neutron-proton interaction into account [26]. In this paper, the improved Nilsson parameters are taken for the PSM calculations of collective rotations of the magnesium isotopes.

\section{THE MODEL}

The details of the PSM can be found in Ref. [21] where the angular-momentum projection was performed within the Nilsson model with BCS pairing. In the present calculation, we improved the pairing calculations by using the LipkinNogami approach [27-29] to avoid the collapse of the BCS pairing method, which can occur in weak pairing due to broken-pair excitations. One can perform the PSM calculation with the mean-field ground state only, without any excited configuration considered. However, it has been pointed out that the inclusion of excited quasiparticle (qp) configurations is important [21], and it is a difficult task to include various qp excitations. In even-even nuclei, they can be two-qp, four-qp, and higher even-number qp configurations, while for odd nuclei, they are one-qp, three-qp, and higher odd-number qp excited configurations. In the present PSM calculation in which we investigate even-even nuclei, lowly excited two-quasiproton and two-quasineutron configurations are considered. 
The configuration space is (for even-even nuclei)

$$
\left\{|0\rangle, a_{v_{i}}^{\dagger} a_{v_{j}}^{\dagger}|0\rangle, a_{\pi_{i}}^{\dagger} a_{\pi_{j}}^{\dagger}|0\rangle, a_{v_{i}}^{\dagger} a_{v_{j}}^{\dagger} a_{\pi_{k}}^{\dagger} a_{\pi_{l}}^{\dagger}|0\rangle\right\},
$$

where $|0\rangle$ is the Nilsson-BCS qp vacuum in which all neutrons and protons are paired giving the lowest energy with zero spin and positive parity, $a_{v_{i}}^{\dagger} a_{v_{j}}^{\dagger}|0\rangle$ and $a_{\pi_{i}}^{\dagger} a_{\pi_{j}}^{\dagger}|0\rangle$ indicate two-quasineutron and two-quasiproton excited configurations, respectively, with two quasiparticles occupying the $i$ th and $j$ th Nilsson orbits, and $a_{v_{i}}^{\dagger} a_{v_{j}}^{\dagger} a_{\pi_{k}}^{\dagger} a_{\pi_{l}}^{\dagger}|0\rangle$ are low-lying four-qp (two quasineutrons and two quasiprotons) configurations. The basis configurations are made within deformed Nilsson single-particle orbits, with the Nilsson deformation parameters taken from Ref. [26]. The deformations, which vary from nucleus to nucleus, are determined by potential-energy-surface calculations [26]. The authors of Ref. [26] suggested new isospin-dependent parameters of the Nilsson potential specially for nuclei in the island of inversion, giving large quadrupole deformations which agree well with observations. We will discuss deformations in more detail later.

The PSM wave functions can be expressed in terms of angular-momentum-projected multi-qp states [21]

$$
\left|\psi^{I}\right\rangle=\sum_{\xi} f_{\xi}^{I} \hat{P}_{M K_{\xi}}^{I}\left|\phi_{\xi}\right\rangle,
$$

where $\left|\phi_{\xi}\right\rangle$ is a basis state given in Eq. (1). $\hat{P}_{M K_{\xi}}^{I}$ is the angular-momentum-projection operator and the coefficient $f_{\xi}^{I}$ is the corresponding weight factor, which is obtained by diagonalizing the eigenvalue equation

$$
\sum_{\xi^{\prime}}\left(H_{\xi^{\prime}}^{I}-E_{I} N_{\xi^{\prime}}^{I}\right) f_{\xi^{\prime}}^{I}=0,
$$

where $H_{\xi \xi^{\prime}}^{I}$ and $N_{\xi \xi^{\prime}}^{I}$ are the matrix elements of the Hamiltonian and the norm, respectively, defined by

$$
H_{\xi \xi^{\prime}}^{I}=\left\langle\phi_{\xi}\left|\hat{H} \hat{P}_{K_{\xi} K_{\xi^{\prime}}^{\prime}}^{I}\right| \phi_{\xi}^{\prime}\right\rangle, \quad N_{\xi \xi^{\prime}}^{I}=\left\langle\phi_{\xi}\left|\hat{P}_{K_{\xi} K_{\xi^{\prime}}^{\prime}}^{I}\right| \phi_{\xi}^{\prime}\right\rangle .
$$

The weight factor $f_{\xi}^{I}$ reflects the mixing amplitudes of different qp configurations. An axially symmetric shape is assumed, and thus each basis state can be labeled by the good quantum number of the spin projection $K$ onto the symmetry axis. The state $\left|\psi^{I}\right\rangle$ is a linear combination of various $K$ states, i.e., $K$ mixing.

The Hamiltonian takes the following form, which includes quadrupole-quadrupole interaction and monopole plus quadrupole pairings [21]:

$\hat{H}=\hat{H}_{0}-\frac{1}{2} \chi \sum_{\lambda} \hat{Q}_{\lambda}^{\dagger} \hat{Q}_{\lambda}-G_{M} \hat{P}^{\dagger} \hat{P}-G_{Q} \sum_{\lambda} \hat{P}_{\lambda}^{\dagger} \hat{P}_{\lambda}$

where $\hat{H}_{0}=\sum_{\alpha} e_{\alpha} c_{\alpha}^{\dagger} c_{\alpha}$ with $e_{\alpha}$ for spherical Nilsson singleparticle levels [21]. The monopole pairing strengths are taken to be $G_{M}=\left[G_{1} \mp G_{2}(N-Z) / A\right] / A$, where the signs "+" and "-_" are for protons and neutrons, respectively, with $G_{1}=$ 18.52 and $G_{2}=11.74$ [30]. The quadrupole pairing strength $G_{Q}$ is assumed to be proportional to $G_{M}$, with a constant factor of 0.40 , i.e., $G_{Q}=0.4 G_{M}$ [30].

\section{CALCULATIONS AND DISCUSSION}

The magic number $N=20$ is well established in stable nuclei [1]. It is normally expected that a closed shell leads to a spherical shape of the nucleus. However, experiments indicate large quadrupole deformations in ${ }^{32,34} \mathrm{Mg}$ [4,31]. We focus on even-even $\mathrm{Mg}$ isotopes with neutron numbers from 12 to 28 .

\section{A. Single-particle orbits in the isospin-dependent Nilsson potential}

With the mean-field parameters obtained by fitting to the nuclear properties near the $\beta$-stability line, a spherical ground state for ${ }^{32} \mathrm{Mg}$ with $N=20$ was obtained [18,32-34], which contradicts with the experimental measurements of a large quadrupole deformation [5,6]. It has been found that the inclusion of the isospin dependence of mean-field potentials can improve the calculations of neutron-rich nuclei $[26,35]$ by providing an effective neutron-proton interaction. The simplest way to include the isospin correlation in the Nilsson model is to consider isospin dependence in the Nilsson parameters. In Ref. [26], the authors suggested a set of isospin-dependent Nilsson parameters which can remarkably improve the calculations of the deformations and binding energies for the ground states of magnesium isotopes.

In the present work, we use the adjusted Nilsson parameters for the $s d$ shell, which are [26]

$$
\begin{aligned}
\kappa_{\pi}=0.105 \times(1-1.1 \times|\mathcal{I}|), & \mu_{\pi}=0.0 \times|\mathcal{I}| \text { for protons, } \\
\kappa_{\nu}=0.105 \times(1+1.1 \times|\mathcal{I}|), & \mu_{\nu}=0.8 \times|\mathcal{I}| \text { for neutrons, }
\end{aligned}
$$

where $\mathcal{I}=(N-Z) / A$, giving isospin dependence, and the parameters $\kappa$ and $\mu$ are the coefficients of the spin-orbit and $\vec{l}^{2}$ terms in the Nilsson potential [25]. The adjusted $\kappa$ and $\mu$ values are close to the standard parameters for nuclei near the line of stability, but they deviate from the standard values when going to the neutron drip line. It is stated in Ref. [26] that the isospin-dependent parameters give large quadrupole deformations for the magnesium isotopes listed in Table I, which is consistent with experimental observations.

In the present PSM calculations, three major shells $(\mathcal{N}=$ $1,2,3)$ for both neutrons and protons are considered; these should be enough for the calculations of low-lying states in the magnesium isotopes. Figure 1 displays the Nilsson levels against the $\varepsilon_{2}$ deformation, calculated at ${ }^{32} \mathrm{Mg}$. It is seen that the adjustments of the Nilsson parameters for the $s d$ shell results in clear shifts of the single-particle levels. Note that no adjustments are needed for other shells [26], e.g., the $f_{7 / 2}$ shell. In Fig. 1(a), we see that the $N=20$ neutron gap is

TABLE I. Deformation parameters $\left(\varepsilon_{2}\right.$ and $\left.\varepsilon_{4}\right)$ used in calculations for $\mathrm{Mg}$ isotopes. They are taken from Ref. [26] for prolate deformations of these nuclei.

\begin{tabular}{cccccccccc}
\hline \hline$N$ & 12 & 14 & 16 & 18 & 20 & 22 & 24 & 26 & 28 \\
\hline$\varepsilon_{2}$ & 0.374 & 0.237 & 0.231 & 0.400 & 0.460 & 0.351 & 0.273 & 0.252 & 0.327 \\
$\varepsilon_{4}$ & 0.069 & 0.026 & 0.048 & 0.024 & 0.066 & 0.018 & -0.004 & 0.044 & 0.062 \\
\hline \hline
\end{tabular}


(a) Neutron

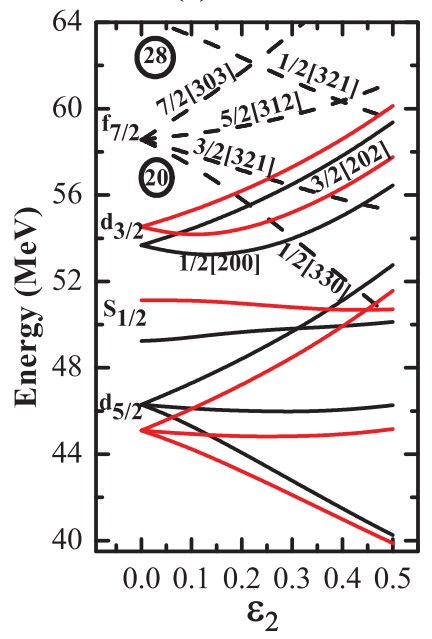

(b) Proton

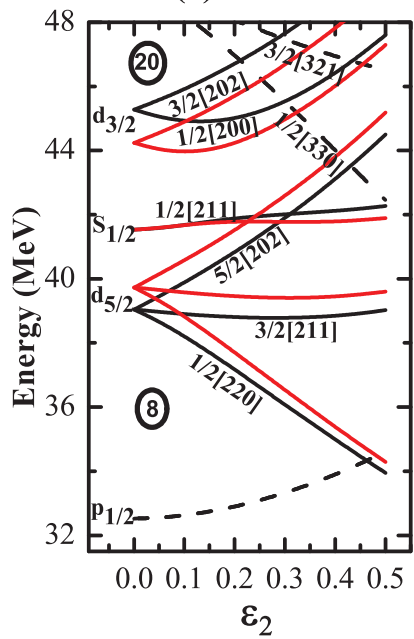

FIG. 1. (Color online) Nilsson diagrams for neutrons (a) and protons (b), calculated at ${ }^{32} \mathrm{Mg}$. The black lines (solid for positive parity and dashed for negative parity) are for single-particle levels calculated by using the standard Nilsson parameters, while the red solid curves denote the $s d$-shell levels (positive parity) obtained using the adjusted parameters [cf. Eq. (6)].

reduced due to the adjustments of the parameters, leading to earlier crossings between the $f_{7 / 2}$ and $d_{3 / 2}$ orbits with increasing deformation. This increases the probabilities of neutrons occupying the $f_{7 / 2}$ intruder orbits, driving nuclei to be more prolate for neutron-rich isotopes of this mass region. For the proton system of magnesium, the variation of the $Z=20$ shell gap has less effect on the properties of the $\mathrm{Mg}$ isotopes because the $Z=12$ Fermi surface is far from the $Z=20$ closed shell. In Ref. [26], it has been clearly shown that the adjusted Nilsson parameters can well describe the ground states of the neutron-rich $\mathrm{Mg}$ isotopes in the island of inversion. In the present work, we adopt the new set of parameters to investigate the collective properties of excited states of these isotopes.

\section{B. Level scheme and electric quadrupole transitions}

We have calculated level schemes for even-even $\mathrm{Mg}$ isotopes by using the PSM; these are shown in Fig. 2. It is seen that the present calculations can reproduce well experimental data, except for the case of ${ }^{30} \mathrm{Mg}$. This exception may be explained by the experimental ratio of $E\left(4_{1}^{+}\right) / E\left(2_{1}^{+}\right)$being 2.3 in ${ }^{30} \mathrm{Mg}$, which is close to the value of 2.5 , the limit of the nonaxial $\gamma$-soft rotor [41]. The nonaxial degree of freedom is not included in the present model.

From Fig. 2, we see that the excitation energies of the $I=2$ states decrease gradually from ${ }^{26} \mathrm{Mg}$ to ${ }^{36} \mathrm{Mg}$, and the excitation energies of the $I=4$ states decrease even faster. This implies that the collectivity increases with increasing neutron number in the $\mathrm{Mg}$ isotopes. This trend is reproduced in our calculations. From the calculated spectra of ${ }^{38} \mathrm{Mg}$, we see that a clear deviation from the collective rotational characteristic occurs at $\operatorname{spin} I=6$. This would indicate a

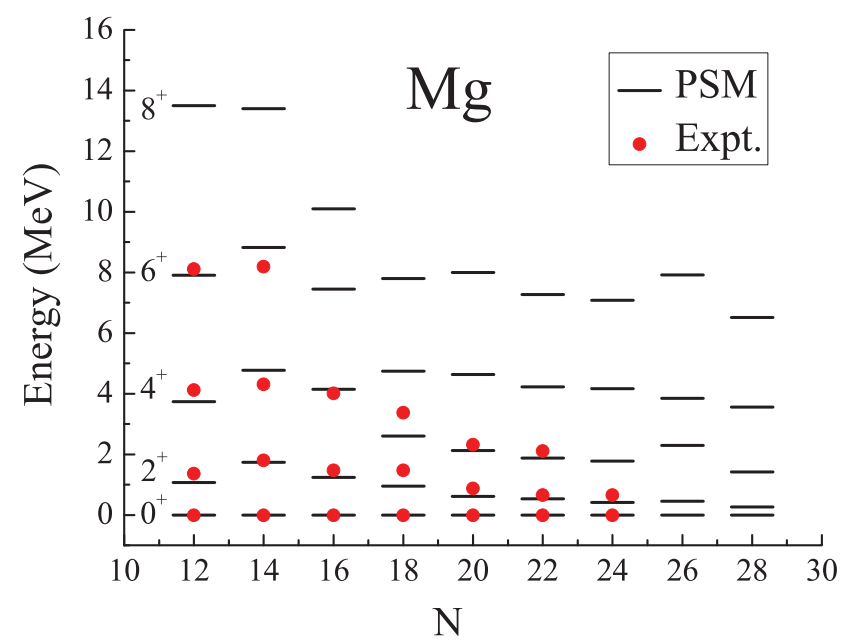

FIG. 2. (Color online) Calculated and experimental spectra of the $\mathrm{Mg}$ isotopes. The experimental data are taken from Refs. [36-40].

structure change, which will be analyzed in more detail in the following section by using the language of band diagrams.

The $B(E 2)$ transition probability is an important observable to probe nuclear structure. In the PSM model, the $B(E 2)$ value from an initial state with spin $I-2$ to a final state at spin $I$ is calculated by [21]

$$
B(E 2 ; I-2 \rightarrow I)=\frac{1}{2 I-3}\left|\left\langle\psi^{I}\left\|e_{\tau_{z}} r^{2} Y_{2}\right\| \psi^{I-2}\right\rangle\right|^{2},
$$

where $\psi^{I}$ is the PSM wave function defined in Eq. (2). The effective charges $e_{\tau_{z}}$ take the standard values $e_{\pi}=1.5 e$ and $e_{v}=0.5 e$ for all $\mathrm{Mg}$ isotopes in the present calculations. The $B(E 2)$ values from the $0^{+}$ground states to the first $2^{+}$ states have been calculated (see Fig. 3) and reproduce well the experimental data. For ${ }^{32} \mathrm{Mg}$, different experimental groups give quite different data. Our calculation supports GANIL data [9], which gives the largest value compared to other groups. The calculated $B(E 2)$ values reach a maximum at

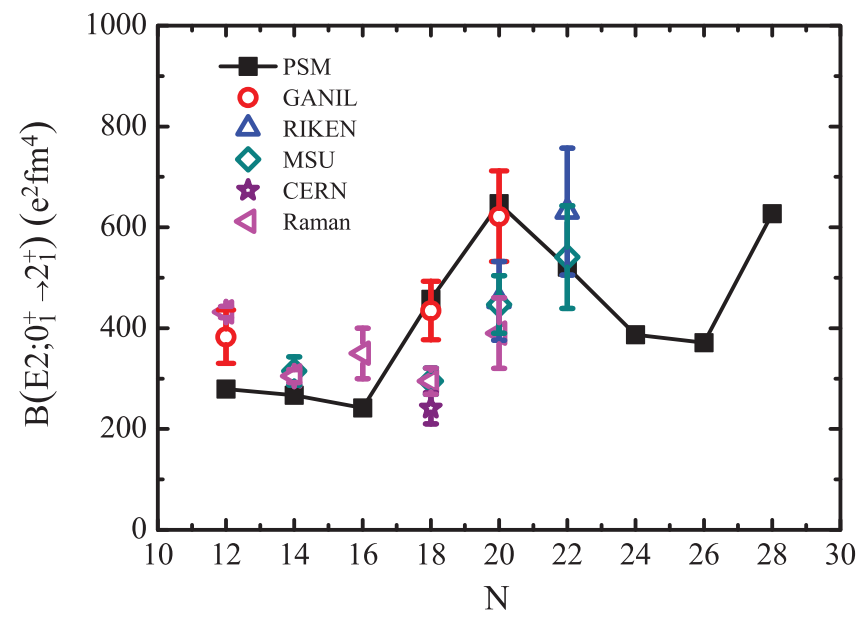

FIG. 3. (Color online) Calculated $B\left(E 2 ; 0_{1}^{+} \rightarrow 2_{1}^{+}\right)$values for the $\mathrm{Mg}$ isotopes. Experimental data from different groups are labeled with GANIL [9], RIKEN [4,31], MSU [8,42], CERN [43], and Raman [44]. 
${ }^{32} \mathrm{Mg}$ in the whole chain of isotopes, which would imply large collectivity and the erosion of the $N=20$ shell gap. Our results are in general consistent with the other theoretical calculations, e.g., the shell model $[12,45]$, the quantum Monte Carlo shell model [13], and the angular-momentum-projected generator coordinate method with a Gogny force [19].

In Ref. [26], $B\left(E 2 ; 0_{1}^{+} \rightarrow 2_{1}^{+}\right)$was calculated by using a phenomenological formula with the quadrupole moment of the ground state. The present microscopic PSM calculations give very similar results to those given in Ref. [26]. This can be easily understood since we are using the same deformations and the same Nilsson single-particle potential as in Ref. [26]. For a well-deformed nucleus, the collective rotation should not change significantly the intrinsic property of the nucleus, at least for the first $2^{+}$state, which implies that the calculated $B\left(E 2 ; 0_{1}^{+} \rightarrow 2_{1}^{+}\right)$value from the PSM wave functions should be close to that from the phenomenological calculation based on the ground-state property.

\section{Band diagrams}

The band diagram, which is the ensemble of different rotational bands plotted in one figure [21], is used to intuitively show the rotational behavior of each multi-qp configuration as well as its relative energy compared to other configurations. From the diagram we can directly learn how these configurations contribute to the structure of yrast states. At a certain spin $I$, the mixing amplitude of a specific quasiparticle configuration, which is described by the weight factor $f_{\xi}^{I}$ [see Eq. (2)], can also be illustrated in the language of the band diagram. A large weight factor indicates a large component (mixing amplitude) of the configuration in the wave function. For example, if a quasiparticle rotational band is close in energy to the yrast line, the mixing amplitude (i.e., weight factor $f_{\xi}^{I}$ ) of the quasiparticle configuration is large in the wave function of the yrast state.

As the deformed basis maintains axial symmetry, we use the quantum number $K$ (the projection of angular momentum onto the symmetry axis of the deformed body) to classify the configurations. For even-even nuclei, the 0-qp band has $K=0$, whereas the $K$ number of the multi-qp band is given by the sum of the quasiparticle $K$ values. The band diagram for a given configuration is defined by [21]

$$
E_{\xi}^{I}=\frac{\left\langle\phi_{\xi}\left|\hat{H} \hat{P}_{K_{\xi} K_{\xi}}^{I}\right| \phi_{\xi}\right\rangle}{\left\langle\phi_{\xi}\left|\hat{P}_{K_{\xi} K_{\xi}}^{I}\right| \phi_{\xi}\right\rangle},
$$

where $\left|\phi_{\xi}\right\rangle$ is a quasiparticle configuration obtained from the Nilsson potential [cf. Eq. (1)].

For the magnesium isotopes investigated, the neutron $d_{3 / 2}$ and $f_{7 / 2}$ and proton $d_{5 / 2}$ and $s_{1 / 2}$ orbits are located near the Fermi surfaces (see Fig. 1). Quasiparticle configurations based on these orbits should be the most important components for low-lying rotational bands. Figure 4 displays the calculated low-lying band diagrams for ${ }^{26-40} \mathrm{Mg}$. For an simple presentation, we only plot the most important configurations in the band diagram. We see that there exists no evident band crossings at $I \leqslant 10$, except for the case of ${ }^{28} \mathrm{Mg}$. For ${ }^{26,30-36} \mathrm{Mg}$, the yrast bands have dominant components of the
0 -qp ground-state bands. With increasing spin, low- $K$ twoquasineutron configurations formed by the intruder orbitals of the $v f_{7 / 2}$ shell start to compete with the 0-qp configuration in the yrast wave functions in ${ }^{30-36} \mathrm{Mg}$, whereas in ${ }^{26} \mathrm{Mg}$ a two-quasiproton configuration formed by the orbitals of the $\pi d_{5 / 2}$ shell plays a role. Strong $K$ mixing occurs in the $I \geqslant 8$ range where different rotational bands have energies close to each other. It was commented that the large-amplitude quadrupole-shape fluctuations would play an important role for the inversion of two low $0^{+}$states in ${ }^{30-34} \mathrm{Mg}$ [46]. As a result of deformations fixed in our calculations, we cannot include the shape fluctuation apparently, but the effect of the dynamical correlation could be partly included by the configuration mixing in the PSM [21,47].

In the band diagrams of ${ }^{28,38,40} \mathrm{Mg}$, evident band crossings and low-energy high- $K$ quasiparticle excitations appear at $I \approx 4-6$. In ${ }^{28} \mathrm{Mg}, K=1$ and 2 bands formed by the two-quasineutron $1 / 2[330] \otimes 3 / 2$ [321] configuration cross with the 0 -qp band (i.e., the ground-state band) at $I \approx 5$ and become lower in energy than the ground-state band. In ${ }^{40} \mathrm{Mg}$, at spin $I=4$ a two-quasineutron $1 / 2[321] \otimes 7 / 2[303]$ configuration appears at an excitation energy close to that of the ground-state band. ${ }^{38} \mathrm{Mg}$ is an interesting nucleus in which our calculations give low-lying multi-qp high- $K$ states with excitation energies lower than that of the ground-state band. In this nucleus, the high- $\Omega$ orbits $7 / 2$ [303] and 5/2[312] that belong to the $f_{7 / 2}$ shell are near the neutron Fermi surface, which can lead to low-energy high- $K$ quasipartcle excitation. The two-qp 7/2[303] $\otimes v 5 / 2$ [312] $K=6$ state appears below the $I^{\pi}=6^{+}$member of the ground-state band [see Fig. $\left.4(\mathrm{~g})\right]$. Due to the high $K$ and low energy, the $K=6$ state would be an isomer. The low-lying high- $K$ multi-qp excitations influence the regularity of the collective rotation of the yrast band in this nucleus. As we shall discuss later, the influence can also be seen in $g$ factors.

By analyzing the band diagrams, we see that the $f_{7 / 2}$ intruder orbitals have significant effects on the low-lying states of $\mathrm{Mg}$ isotopes within the island of inversion. The interplay between normal and intruder configurations is embodied in the present model through the mixing of different multiquasiparticle configurations, which is similar to some extent to the shell-model calculations.

\section{Gyromagnetic factors}

The $g$ factor which reflects the magnetic property of a state is a sensitive probe of the configuration. It is defined by

$$
g(I)=\frac{\mu(I)}{\mu_{N} I}=\frac{1}{\mu_{N} I}\left[\mu_{\pi}(I)+\mu_{v}(I)\right],
$$

where $\mu_{N}$ is the nuclear magneton and $\mu_{\tau}(I)$ is the magnetic moment of a state $\Psi^{I}$, obtained by [48]

$$
\begin{aligned}
\mu_{\tau}(I)= & \frac{I}{\sqrt{I(I+1)}}\left\langle\Psi^{I}\left\|\hat{\mu}^{\tau}\right\| \Psi^{I}\right\rangle \\
= & \frac{I}{\sqrt{I(I+1)}}\left[g_{l}^{\tau}\left\langle\Psi^{I}\left\|\hat{j}^{\tau}\right\| \Psi^{I}\right\rangle\right. \\
& \left.+\left(g_{s}^{\tau}-g_{l}^{\tau}\right)\left\langle\Psi^{I}\left\|\hat{s}^{\tau}\right\| \Psi^{I}\right\rangle\right],
\end{aligned}
$$




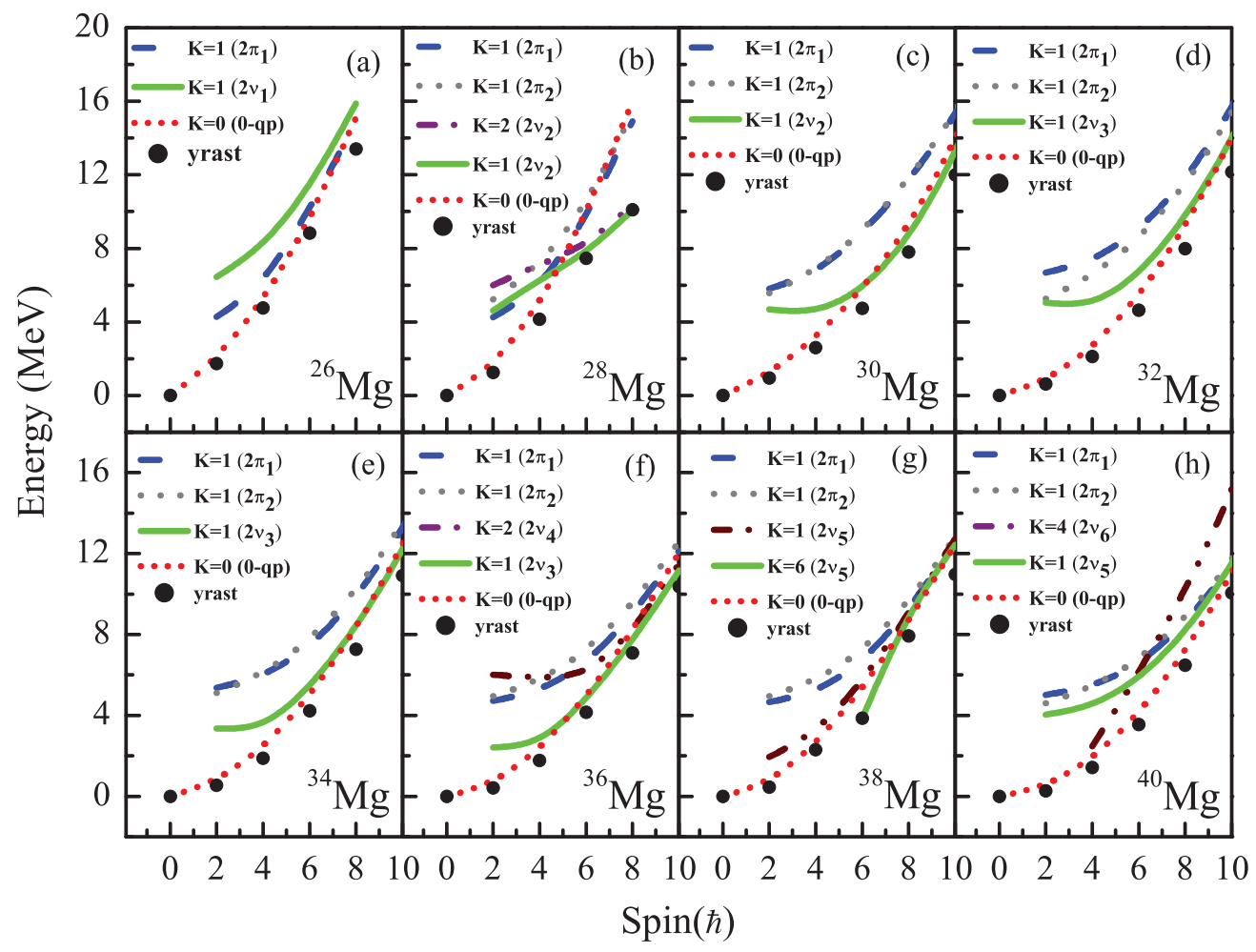

FIG. 4. (Color online) Calculated low-lying band diagrams for Mg isotopes. The configurations are $\left\{2 \pi_{1}: \pi \frac{3}{2}[211] \otimes \pi \frac{5}{2}[202]\right\}$, $\left\{2 \pi_{2}\right.$ : $\left.\pi \frac{3}{2}[211] \otimes \pi \frac{1}{2}[211]\right\},\left\{2 v_{1}: v \frac{3}{2}[211] \otimes v \frac{5}{2}[202]\right\},\left\{2 v_{2}: v \frac{1}{2}[330] \otimes v \frac{3}{2}[321]\right\},\left\{2 v_{3}: v \frac{3}{2}[321] \otimes v \frac{5}{2}[312]\right\},\left\{2 v_{4}: v \frac{1}{2}[321] \otimes v \frac{5}{2}[312]\right\},\left\{2 v_{5}:\right.$ $\left.v \frac{5}{2}[312] \otimes v \frac{7}{2}[303]\right\}$, and $\left\{2 v_{6}: v \frac{1}{2}[321] \otimes v \frac{7}{2}[303]\right\}$.

where $\tau=\pi$ or $v$ denotes protons or neutrons, respectively. In our calculation, the standard values for $g_{l}$ and $g_{s}$ are taken as

$$
\begin{array}{ll}
g_{l}^{\pi}=1, & g_{s}^{\pi}=5.586 \times 0.75, \\
g_{l}^{\nu}=0, & g_{s}^{\nu}=-3.826 \times 0.75 .
\end{array}
$$

The neutron alignment of one pair of neutrons results in a more negative value for the $g$ factor, while the proton alignment leads to an increase of the $g$ factor.

The calculated $g$ factors for the yrast states of the $\mathrm{Mg}$ isotopes compared with experimental data [49] are shown in Fig. 5. We see that the $g$ factor is a good indicator of the configuration. In ${ }^{28} \mathrm{Mg}$ [see Fig. 4(b) for its band diagram], the

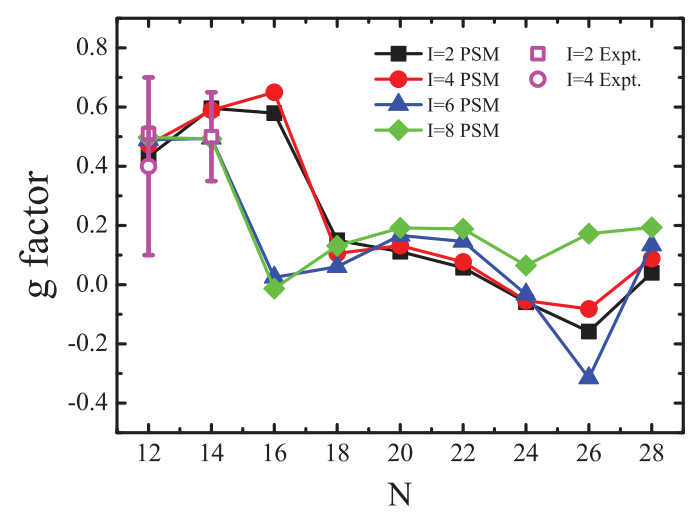

FIG. 5. (Color online) Calculated $g$ factors for the yrast states of $\mathrm{Mg}$ isotopes compared to data [49]. two-quasineutron $1 / 2[330] \otimes 3 / 2[321]$ bands with $K=1$ and 2 cross the ground-state band at spin $I \approx 5$, and they become dominant components in the yrast band after the crossing. This corresponds to a sudden drop at $I=6$ and 8 for the $g$ factors in Fig. 5. For ${ }^{30-36} \mathrm{Mg}$, the $g$-factor values are close to zero. Compared with the lighter isotopes ${ }^{24-28} \mathrm{Mg}$, the small $g$-factor values in ${ }^{30-36} \mathrm{Mg}$ imply an increase of components of neutron configurations in the yrast bands. This would indicate shell erosion around $N=20$, which is consistent with the indication from the $B(E 2)$ evolution, shown in Fig. 3. The weakening of the $N=20$ shell gap increases the ease of producing quasineutron excitations. An abrupt drop of the $g$ factor occurs at $I=6$ in ${ }^{38} \mathrm{Mg}$. This can be explained from the band diagram in Fig. 4(g) in which we see that a two-quasineutron $K=6$ state appears below the 0-qp ground-state band and dominates in the yrast band. These $g$ factors can supply important information about the structures of the yrast states. Further experiments are needed for the predictions.

\section{E. The $K^{\pi}=6^{+}$band}

The above analysis of band diagrams and $g$ factors points to the possible existence of a low-lying high- $K$ isomeric state in ${ }^{38} \mathrm{Mg}$. For an isomeric state, besides excitation energy, the electric quadrupole transition is another interesting and useful observable, particularly for transitions into low- $K$ states, which would be highly hindered. Figure 6 shows the calculated energies of the predicted $K^{\pi}=6^{+}\left(\nu \frac{5}{2}[312] \otimes \nu \frac{7}{2}[303]\right)$ band, 


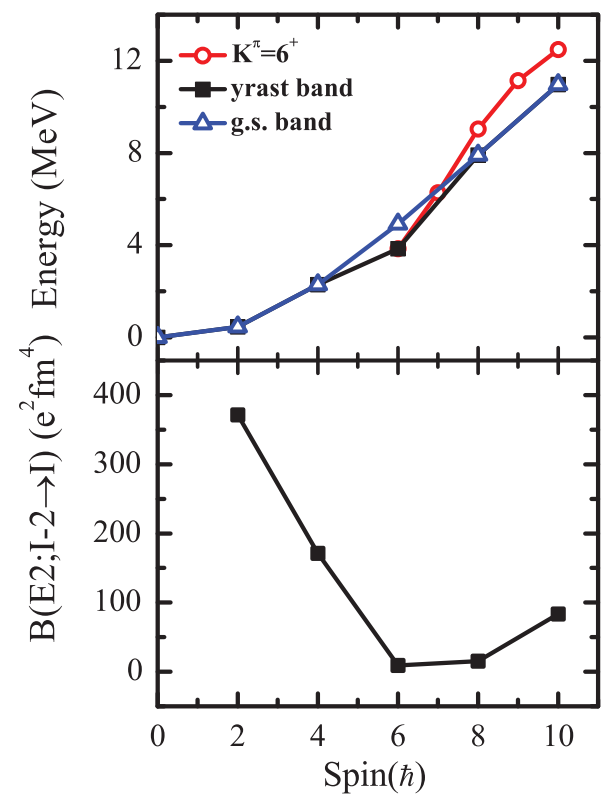

FIG. 6. (Color online) Predicted energies of ground-state, yrast, and $K^{\pi}=6^{+}\left(v \frac{5}{2}[312] \otimes v \frac{7}{2}[303]\right)$ bands for ${ }^{38} \mathrm{Mg}$ (upper panel) and calculated $B(E 2)$ values along the yrast line (lower panel).

compared with ground-state and yrast bands, and the $B(E 2)$ values along the yrast line for ${ }^{38} \mathrm{Mg}$. It is seen that the $K^{\pi}=6^{+}$ state appears at an energy lower than the $I^{\pi}=6^{+}$member of the ground-state band, and it becomes the yrast state actually. Calculated $B(E 2)$ values between the $K^{\pi}=6^{+}$state and the $I^{\pi}=4^{+}, 8^{+}$members of the ground-state band are much smaller than the in-band $E 2$ transitions of the ground-state band, showing high hindrance for transitions from high- $K$ to low- $K$ states. The low excitation energy and low transition rate lead to the prediction that the $K^{\pi}=6^{+}$state would be an isomer.

\section{F. The second $0^{+}$states}

It was pointed out that shape coexistence would be evident in nuclei around the island of inversion [50], mainly in ${ }^{30,32,34} \mathrm{Mg}$ for magnesium isotopes. Nowadays the investigation of the shapes of the first and second $0^{+}$states is still an interesting topic from both theoretical [12,13,19,46,51] and experimental [52-54] viewpoints. As the fluctuation of different intrinsic shapes is not considered in the present framework, we analyze qualitatively $0^{+}$states which appear at different deformations through the expectation value of the PSM Hamiltonian,

$$
E_{0}\left(\varepsilon_{2}\right)=\frac{\left\langle\varepsilon_{2}|\hat{H}| \varepsilon_{2}\right\rangle}{\left\langle\varepsilon_{2} \mid \varepsilon_{2}\right\rangle},
$$

where $\hat{H}$ is the Hamiltonian as given in Eq. (5), and $\left|\varepsilon_{2}\right\rangle \equiv|0\rangle$ is the BCS qp vacuum at deformation $\varepsilon_{2}$. The calculated results for ${ }^{30,32,34} \mathrm{Mg}$ are shown in Fig. 7. We see shape coexistence of the spherical and the deformed $0^{+}$states in ${ }^{30} \mathrm{Mg}$, which is consistent with experimental observation of the small electric monopole transition $B\left(E 0 ; 0_{2}^{+} \rightarrow 0_{1}^{+}\right)$[53]. It is also seen that deformed $0^{+}$states become more favored energetically than

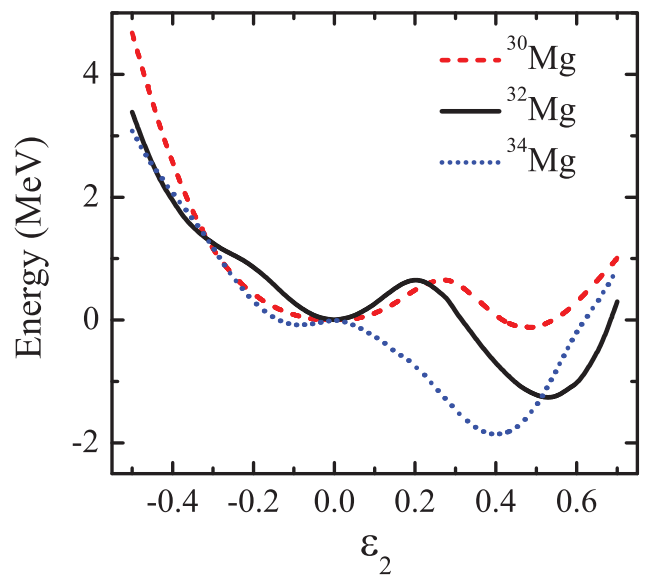

FIG. 7. (Color online) Variation of the energy $E_{0}\left(\varepsilon_{2}\right)$ [defined by Eq. (12)] with deformation $\varepsilon_{2}$. In the plot, the energy of the spherical state is set at zero.

the spherical state in ${ }^{32,34} \mathrm{Mg}$, making deformed and spherical $0^{+}$states reversed in energy, which leads to the phenomenon of the island of inversion.

\section{SUMMARY}

In this paper, based on the new set of isospin-dependent Nilsson parameters, we have employed the projected shell model for a systematical investigation of the collectivity of excited states for neutron-rich magnesium isotopes within the island of inversion. To avoid the BCS pairing collapse, we have improved the pairing treatment by using the Lipkin-Nogami approach for the PSM calculations.

The calculated excitation energies of low-lying states reproduce well the experimental data. Calculated $E 2$ transition probabilities from the $0^{+}$ground states to the first $2^{+}$states in ${ }^{24-40} \mathrm{Mg}$ are in reasonable agreement with experimental measurements. For nuclei around ${ }^{32} \mathrm{Mg}$, we obtained large $B(E 2)$ values, which would indicate erosion of the $N=20$ shell gap. This is consistent with some other theoretical calculations.

By analyzing the band diagrams, we have found that, for ${ }^{28,38} \mathrm{Mg}$, two-quasineutron configurations formed by the orbitals of the $v f_{7 / 2}$ shell dominate in the yrast states at spins $I \geqslant 6$, which changes the collective rotational regularity of the yrast bands. Especially for ${ }^{38} \mathrm{Mg}$, a two-quasineutron $5 / 2[312] \otimes 7 / 2[303] K^{\pi}=6^{+}$state with a low excitation energy was predicted to be an isomer. The neutron $f_{7 / 2}$ intruder orbitals have significant effects on the low-lying states of $\mathrm{Mg}$ isotopes within the island of inversion. As we have seen, these effects occur in the $g$ factors. The small nearly zero $g$-factor values in ${ }^{30-36} \mathrm{Mg}$ imply an increase of components of neutron configurations in the yrast bands, compared with the lighter isotopes ${ }^{24-28} \mathrm{Mg}$. This would indicate shell erosion around $N=20$. For ${ }^{28} \mathrm{Mg}$, the $g$ factor drops abruptly at $I=6$, which is explained by band crossings between two-quasineutron and ground-state bands. Existing experimental $g$ factors have been reproduced well, and more measurements of $g$ factors for neutron-rich $\mathrm{Mg}$ isotopes are desired. 


\section{ACKNOWLEDGMENTS}

We thank C. F. Jiao for valuable discussions. This work has been supported by the National Key Basic Research Program of China under Grant No. 2013CB834400 and the
National Natural Science Foundation of China under Grants No. 11235001 and No. 11205120. X. B. Wang acknowledges support from the University of Jyväskylä within the FIDIPRO programme.
[1] O. Sorlin and M. G. Porguet, Prog. Part. Nucl. Phys. 61, 602 (2008).

[2] C. Thibault et al., Phys. Rev. C 12, 644 (1975).

[3] X. Campi, H. Flocard, A. K. Kerman, and S. Koonin, Nucl. Phys. A 251, 193 (1975).

[4] T. Motobayash et al., Phys. Lett. B 346, 9 (1995).

[5] D. Guillemaud-Muller et al., Nucl. Phys. A 426, 37 (1984).

[6] S. Takeuchi et al., Phys. Rev. C 79, 054319 (2009).

[7] D. T. Yordanov et al., Phys. Rev. Lett. 108, 042504 (2012).

[8] B. V. Pritychenko et al., Phys. Lett. B 461, 322 (1999).

[9] V. Chisté et al., Phys. Lett. B 514, 233 (2001).

[10] J. R. Terry et al., Phys. Rev. C 77, 014316 (2008).

[11] E. K. Warburton, J. A. Becker, and B. A. Brown, Phys. Rev. C 41, 1147 (1990).

[12] E. Caurier, F. Nowacki, A. Poves, and J. Retamosa, Phys. Rev. C 58, 2033 (1998).

[13] Y. Utsuno, T. Otsuka, T. Mizusaki, and M. Honma, Phys. Rev. C 60, 054315 (1999).

[14] T. Otsuka, R. Fujimoto, Y. Utsuno, B. A. Brown, M. Honma, and T. Mizusaki, Phys. Rev. Lett. 87, 082502 (2001).

[15] T. Otsuka, Y. Utsuno, T. Mizusaki, and M. Honma, Nucl. Phys. A 685, 100 (2001).

[16] E. Caurier, G. Martiez-Pinedo, F. Nowacki, A. Poves, and A. P. Zuker, Rev. Mod. Phys. 77, 427 (2005).

[17] F. Maréchal et al., Phys. Rev. C 72, 044314 (2005).

[18] J. Terasaki, H. Flocarda, P. H. Heenen, and P. Bonche, Nucl. Phys. A 621, 706 (1997).

[19] R. Rodríguez-Guzmán, J. L. Egido, and L. M. Robledo, Nucl. Phys. A 709, 201 (2002).

[20] Z. Z. Ren, Z. Y. Zhu, Y. H. Cai, and G. O. Xu, Phys. Lett. B 380, 241 (1996)

[21] K. Hara and Y. Sun, Int. J. Mod. Phys. E 04, 637 (1995).

[22] T. Otsuka, Nucl. Phys. A 722, C347 (2003).

[23] T. Otsuka, T. Suzuki, R. Fujimoto, H. Grawe, and Y. Akaishi, Phys. Rev. Lett. 95, 232502 (2005).

[24] V. I. Isakov, K. I. Erokhina, H. Mach, M. Sanchez-Vega, and B. Fogelberg, Eur. Phys. J. A 14, 29 (2002).

[25] S. G. Nilsson, Mat. Fys. Medd. K. Dan. Vidensk. Selsk. 29, 16 (1955).

[26] Q. J. Zhi and Z. Z. Ren, Phys. Lett. B 638, 166 (2006).
[27] H. C. Pradhan, Y. Nogami, and J. Law, Nucl. Phys. A 201, 357 (1973).

[28] W. Nazarewicz, M. A. Riley, and J. D. Garrett, Nucl. Phys. A 512, 61 (1990).

[29] P. Möller and J. R. Nix, Nucl. Phys. A 536, 20 (1992).

[30] Y. C. Yang, Y. Sun, K. Kaneko, and M. Hasegawa, Phys. Rev. C 82, 031304(R) (2010).

[31] H. Iwasaki et al., Phys. Lett. B 522, 227 (2001).

[32] P. Möller, J. R. Nix, W. D. Myers, and W. J. Swiatecki, At. Data Nucl. Data Tables 59, 185 (1995).

[33] G. A. Lalazissis, A. R. Farhan, and M. M. Sharma, Nucl. Phys. A 628, 221 (1998).

[34] M. V. Stoitsov, J. Dobaczewski, P. Ring, and S. Pittel, Phys. Rev. C 61, 034311 (2000).

[35] M. M. Sharma, G. Lalazissis, J. König, and P. Ring, Phys. Rev. Lett. 74, 3744 (1995).

[36] P. M. Endt, Nucl. Phys. A 633, 1 (1998).

[37] R. B. Firestone, Nucl. Data Sheets 108, 2319 (2007).

[38] M. S. Basunia, Nucl. Data Sheets 111, 2331 (2010).

[39] C. Ouellet and B. Singh, Nucl. Data Sheets 112, 2199 (2011).

[40] A. Gade et al., Phys. Rev. Lett. 99, 072502 (2007).

[41] P. Ring and P. Schuck, The Nuclear Many-body Problem (Springer, Berlin, 1980).

[42] J. A. Church et al., Phys. Rev. C 72, 054320 (2005).

[43] O. Niedermaier et al., Phys. Rev. Lett. 94, 172501 (2005).

[44] S. Raman, C. W. Nestor, Jr., and P. Tikkanen, At. Data Nucl. Data Tables 78, 1 (2001).

[45] J. M. Cook, T. Glasmacher, and A. Gade, Phys. Rev. C 73, 024315 (2006).

[46] N. Hinohara, K. Sato, K. Yoshida, T. Nakatsukasa, M. Matsuo, and K. Matsuyanagi, Phys. Rev. C 84, 061302(R) (2011).

[47] J. A. Sheikh, Y. Sun, and P. M. Walker, Phys. Rev. C 57, R26 (1998).

[48] Y. X. Liu, Y. Sun, X. H. Zhou, Y. H. Zhang, S. Y. Yu, Y. C. Yang, and H. Jin, Nucl. Phys. A 858, 11 (2011).

[49] N. J. Stone, At. Data Nucl. Data Tables 90, 75 (2005).

[50] K. Heyde and J. L. Wood, J. Phys. G 17, 135 (1991).

[51] T. Otsuka, Eur. Phys. J. A 20, 69 (2004).

[52] H. Mach et al., Eur. Phys. J. A 25, 105 (2005).

[53] W. Schwerdtfeger et al., Phys. Rev. Lett. 103, 012501 (2009).

[54] K. Wimmer et al., Phys. Rev. Lett. 105, 252501 (2010). 